\title{
Hypofrontality in men with first-episode psychosis
}

\author{
V. MOLINA, J. SANZ, S. REIG, R. MARTÍNEZ, F. SARRAMEA, R. LUQUE, \\ C. BENITO, J. D. GISPERT, J. PASCAU and M. DESCO
}

\section{Background Decreased metabolic activity in the prefrontal cortex during cognitive activation is a recurrent finding and a likely functional marker of schizophrenia.}

Aims To investigate the occurrence of hypofrontality in patients with firstepisode psychosis, with or without evolution to schizophrenia.

Method We used fluorodeoxyglucose positron emission tomography during the performance of an attention task and magnetic resonance imaging to study the dorsolateral prefrontal region in 13 men with a first episode of psychosis. Data from patients who progressed to schizophrenia were compared with those of patients who did not meet criteria for this diagnosis after 2 years.

\section{Results Patients who developed schizophrenia demonstrated a significant hypofrontality in the dorsolateral prefrontal cortex in comparison with the non-schizophrenia and control groups.}

Conclusions Our results suggest that hypofrontality could be a marker of schizophrenia at the time of the first psychotic episode, in agreement with neurodevelopmental theories of schizophrenia.

Declaration of interest None. Funding detailed in Acknowledgements.
Decreased metabolic activity in the prefrontal cortex during cognitive activation is a recurrent finding in schizophrenia (Volz et al, 1999). If it were not present in other psychotic disorders, hypofrontality could constitute a distinctive marker for schizophrenia. Although other groups have published data on hypofrontality in schizophreniform disorders (Andreasen et al, 1997), we know of none comparing metabolic patterns in these patients in the light of whether or not they progress to schizophrenia. To confirm hypofrontality as a feature typical of schizophrenia, it would be advisable to study patients in very early stages of the disease. This design requires a corroboration of the diagnosis of schizophrenia by a prospective follow-up. With this goal, we used magnetic resonance imaging (MRI) and $\left[{ }^{18} \mathrm{~F}\right]$ fluorodeoxyglucose (FDG) positron emission tomography (PET) to investigate two groups of people with first-episode psychosis: one group in which the diagnosis of schizophrenia was confirmed 2 years later, and a group who did not meet the criteria for this diagnosis after the same period.

\section{METHOD}

\section{Study participants}

The existence of gender-related differences in frontal activity cannot be ruled out, considering that structural alterations seem to be more pronounced in men (Nopoulos et al, 1997). For this reason, and to achieve maximum sample homogeneity, our study was limited to men. Thirteen right-handed men with a first psychotic episode were enrolled in a diachronic study of first-break schizophrenia; all of them were enrolled while hospitalised for the first time in the psychiatric unit of a general hospital. The PET and MRI scans were performed at the time of inclusion in the study, before any of the patients had met the criteria for schizophrenia. To be included in the protocol, patients must have presented with first-episode psychosis with symptoms lasting more than 1 week, not attributable to organic or toxic origin, and unrelated to any other Axis I disorder; the presence of any relevant stressors clearly related to the episode had to be ruled out. We used these criteria in order to avoid recruiting patients with transient psychotic symptoms, according to DSM-IV criteria (American Psychiatric Association, 1994).

After inclusion, all patients were followed for 2 years on an out-patient basis with monthly visits, to confirm or rule out a diagnosis of schizophrenia at the end of this period. After this follow-up, two experienced psychiatrists (V.M. and J.S.), masked to the results of the PET scans, diagnosed each patient and decided whether the index episode was a first break of schizophrenia or, instead, a single psychotic episode that did not evolve into schizophrenia 2 years after the enrolment. For this final diagnosis they used all the available follow-up information (including total duration of significant symptoms), a semi-structured interview, the Structured Clinical Interview for DSM-IV Access I Disorders - Clinician Version (SCID-CV; First et al, 1997) and the information provided by the families and clinical personnel. For diagnostic purposes, the duration of symptoms was calculated covering the period before and after initiation of treatment.

On the basis of their final diagnosis, the 13 patients were divided into two groups. The schizophrenia group comprised six people with a diagnosis of paranoid schizophrenia according to DSM-IV criteria. The non-schizophrenia group comprised six people with a diagnosis of schizophreniform psychosis and one person who had experienced a brief psychotic disorder (with a symptom duration of 3 weeks), likewise according to DSM-IV criteria. The latter diagnoses meant that these patients' psychotic episodes were shorter than required to qualify as schizophrenia, no other psychotic episode occurred during follow-up and the patients did not meet schizophrenia criteria (including enduring negative symptoms) at the end of follow-up. The duration of the disease in the non-schizophrenia group was less than 3 months in all cases. The clinical scores calculated using the Positive and Negative Syndrome Scale (PANSS; Kay et al, 1992) at the time of admission and at the end of follow-up are shown in Table 1. 
Table I Demographic and clinical characteristics of participants

\begin{tabular}{|c|c|c|c|}
\hline & $\begin{array}{l}\text { Schizophrenia } \\
\text { group }(n=6) \\
\text { Mean (s.d.) }\end{array}$ & $\begin{array}{c}\text { Non-schizophrenia } \\
\text { group }(n=7) \\
\text { Mean (s.d.) }\end{array}$ & $\begin{array}{c}\text { Control } \\
\text { group }(n=8) \\
\text { Mean (s.d.) }\end{array}$ \\
\hline Age, years & $24.2(4.4)$ & $29.1(8.8)$ & $28.7(6.0)$ \\
\hline Education, years & II.5 (4.6) & $10.9(6.0)$ & II.8 (4.9) \\
\hline Duration of disease, months before inclusion & I.I (0.8) & $0.9(0.6)$ & \\
\hline Parental socio-economic level & $2.1(1.8)$ & $2.2(1.8)$ & $2.0(1.6)$ \\
\hline \multicolumn{4}{|l|}{ PANSS score } \\
\hline \multicolumn{4}{|l|}{ Positive symptoms } \\
\hline Enrolment & $19.9(5.1)$ & $20.3(6.1)$ & \\
\hline Follow-up & $10.0(3.4)$ & $7.2(2.3)$ & \\
\hline \multicolumn{4}{|l|}{ Negative symptoms } \\
\hline Enrolment & $15.6(6.3)$ & $12.9(7.5)$ & \\
\hline Follow-up & $20.6(8.3)$ & $7.4(4.1)$ & \\
\hline \multicolumn{4}{|l|}{ General symptoms } \\
\hline Enrolment & $33.4(7.1)$ & $30.5(8.8)$ & \\
\hline Follow-up & $30.1(9.5)$ & $17.4(7.7)$ & \\
\hline
\end{tabular}

PANSS, Positive and Negative Syndrome Scale.

All patients were antipsychotic-naïve prior to the presenting episode; they were treated solely during the 2 days prior to the PET scan. This was a practical means of enrolling a sample representative of the usual presentation of schizophrenia, avoiding a selection bias related to the impaired ability of patients with acute psychosis to cooperate during neuroimaging procedures. All patients received the same treatment (haloperidol $10 \mathrm{mg}$ per day liquid formulation) and the medication was discontinued for $12 \mathrm{~h}$ prior to the PET scan. No other treatment was administered before scanning. One patient from the schizophrenia group and two from the non-schizophrenia group had received benzodiazepines for insomnia before inclusion, but they had iscontinued this medication for more than 2 weeks. No acute dystonic reaction occurred, thus there was no need to administer anticholinergic medication before scanning. Nursing personnel verified treatment compliance.

Patients in the schizophrenia group were maintained on antipsychotic treatment for the entire follow-up period. In the non-schizophrenia group, the antipsychotic treatment was continued for a period of 6-12 months following the PET scan, on the basis of clinical criteria. None of the patients in the non-schizophrenia group presented clinically relevant negative symptoms, disorganisation or persistent behavioural alterations at the end of the follow-up period (i.e. more than a year after completing the treatment). The period of complete remission of these symptoms was greater than 1 year in all cases.

Eight healthy men were also recruited as a control reference group. To match them with the patient group, none had an education to college level. No difference in age or parental socio-economic level (Hollingshead \& Frederick, 1953) was observed between the groups (Table 1).

Exclusion criteria for patients and controls were learning disability, neurological disease, MRI findings deemed clinically relevant (from a neurological perspective) by a radiologist masked to the diagnosis, history of head trauma with loss of consciousness, and history of substance misuse during the past 6 months (other than occasional hypnotics). A urinalysis was done in all cases to rule out a toxic cause of the psychotic episode.

After they had been given full information, an informed consent form was signed by the participants and their relatives. The independent ethics committee approved the study.

\section{Image analysis}

To determine the metabolic activity in the dorsolateral prefrontal cortex and the occipital lobe, we used a procedure based on MRI/PET image fusion. This method uses the anatomical information of the MRI to allow detailed measurement of regional metabolic activity in the PET image (Molina et al, 2003).

\section{MRI protocol}

Magnetic resonance images were acquired on a Philips Gyroscan 1.5 T scanner (Philips Medical Systems, Reigate, UK) using a $T_{1^{-}}$ weighted three-dimensional gradient echo sequence with the following parameters: matrix size $256 \times 256$, pixel size $0.9 \mathrm{~mm} \times$ $0.9 \mathrm{~mm}$ (field of view approximately $256 \times 256 \mathrm{~mm}$ ), flip angle $30^{\circ}$, echo time $4.6 \mathrm{~ms}$, slice thickness $1.1-1.5 \mathrm{~mm}$. In addition, $T_{2}$-weighted sequences were acquired for tissue segmentation and for other clinical purposes (turbo spin echo, turbo factor 15 , echo time $120 \mathrm{~ms}$, matrix size $256 \times 256$, slice thickness $5.5 \mathrm{~mm}$ ).

\section{PET protocol}

The PET study used a Siemens Exact 47 tomograph (Siemens, Erlangen, Germany). Scans were obtained $20 \mathrm{~min}$ after injecting $370 \mathrm{MBq}$ of $\left[{ }^{18} \mathrm{~F}\right] \mathrm{FDG}$, while the participant performed a contingent continuous performance test (Rosvold et al, 1956). Participants were instructed to push a button if a letter $\mathrm{T}$ immediately followed the letter $\mathrm{L}$, presented on a computer screen. The interstimulus interval was $1 \mathrm{~s}$. During the PET scan a neuropsychologist supervised the appropriate response and full compliance with the performance test of all participants. After an intravenous line had been inserted for FDG administration, the participant began the task, which was divided into four blocks of $5 \mathrm{~min}$ each, with a $1 \mathrm{~min}$ rest between blocks; FDG was administered 1 min after initiating the task. Participants were instructed to fast for at least $6 \mathrm{~h}$ before the scan, with particular emphasis on avoiding coffee and other psychoactive beverages.

Tracer activity values were proportionally normalised to the global activity of each scan (Frackowiak et al, 1997) thus representing relative activity. Total metabolic activity for each region of interest was divided by the region's volume, thus providing a measurement independent of the amount of tissue sampled.

\section{Segmentation}

To obtain metabolic measurements of the prefrontal cortex and occipital lobe, we used a method for semi-automated segmentation of the brain based on the Talairach reference system, similar to that described 


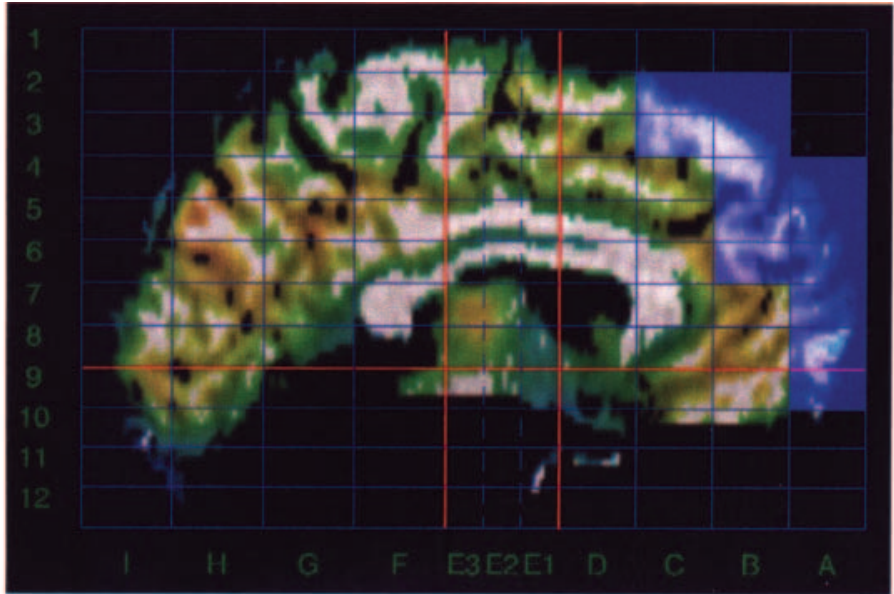

(a)

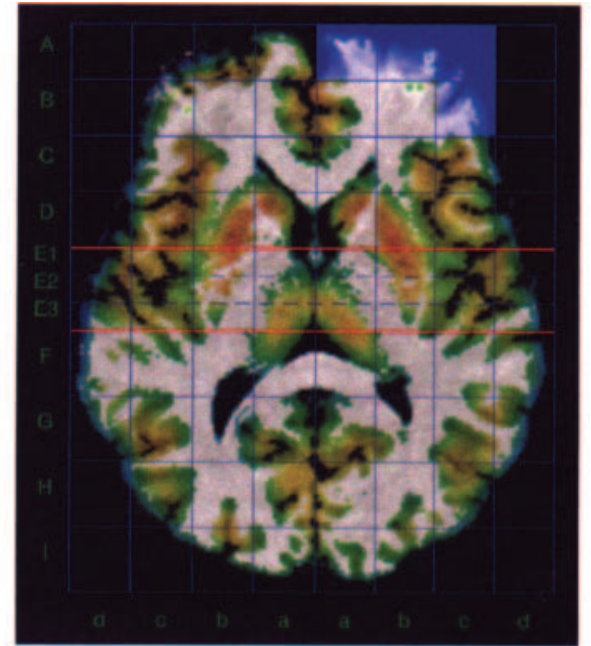

(b)

Fig. I Sagittal (a) and axial (b) views illustrating a Talairach grid built upon an edited magnetic resonance scan and fused with the grey matter segmentation of the positron emission tomographic scan. The Talairach grid cells describing the left dorsolateral prefrontal region are highlighted in blue. This region of interest is defined as the cortex encompassed in Brodmann areas 8, 9, 10 and 46, according to the Talairach atlas (Talairach \& Tournoux, 1988).

by Andreasen et al (1996) and Kates et al (1999). We used a two-step procedure (Desco et al, 2001): the first step involved editing the MRI to remove skull and extracranial tissue, registration of the PET and MRI scans, and an initial segmentation of cerebral tissues into grey matter, white matter and cerebrospinal fluid; in the second stage we applied the Talairach reference system (Talairach \& Tournoux, 1988) to define regions of interest and to obtain final metabolic activity data (Fig. 1).

The edited MRI (without extracranial tissue) was co-registered with the PET image using the Automated Image Registration (AIR) algorithm (Woods et al, 1993). Fusion results were visually checked in all cases and the observed co-registration was always optimal. An initial segmentation of cerebral tissue was performed using an automated method, currently included as a standard processing tool in the Statistical Parametric Mapping (SPM) program (Ashburner \& Friston, 1997). This method classifies all MRI pixels into four tissue types: grey matter, white matter, cerebrospinal fluid and 'other tissues'. The algorithm also removes the effect of radiofrequency field inhomogeneities (Ashburner \& Friston, 1997). This segmentation was checked for inconsistencies and manually corrected whenever necessary by an experienced radiologist masked to diagnosis.

In the second stage, the regions of interest were obtained by superimposing the three-dimensional tissue masks corresponding to the first three tissue types on to each individual's Talairach reference grid (Fig. 1), where the regions of interest were defined as sets of cells. On the MRI/PET fused images, volume and activity were measured by summing the data from the grid cells associated with each region of interest (Desco et al, 2001).

Validity of the Talairach-based procedure as an automated segmentation tool suitable for schizophrenia research has been proved (Andreasen et al, 1996; Kates et al, 1999). A single operator performed all manual procedures, thus avoiding any potential interrater variability. Reliability of the entire segmentation procedure had been previously assessed by repeating the measurements three times for 15 cases selected at random. Repeatability of metabolic activity values ranged from $93 \%$ to 99\% (Molina et al, 2003).

Region of interest variables included in the analysis were the dorsolateral prefrontal cortex and the occipital lobe, which was used only as a reference structure (see below). The dorsolateral prefrontal cortex was defined as the cortex encompassing Brodmann areas 8, 9, 10 and 46. The occipital lobe was defined according to boundaries previously established by other authors also using the Talairach method (Andreasen et al, 1996; Kates et al, 1999). We studied this brain region as a control for potential global effect bias in our measurements of metabolic activity due to the short-course treatment (Bartlett et al,
1994). Data from the occipital cortex allowed us to discard a potential difference in overall metabolic activity between patient groups. The absence of bias due to a global effect was confirmed by the similar values of metabolic activity in the occipital region found in all the groups studied.

Since there were slight age differences between groups, we used Spearman's correlation coefficients to discard a significant effect of age on activity data. None of the correlation coefficients between age and metabolic activity variables was statistically significant.

\section{Statistical analysis}

The study hypothesis was approached by comparing the relative activity in both the right and left dorsolateral prefrontal cortex in the three groups: the schizophrenia patient group, the non-schizophrenia patient group and the control group. Because of the small sample sizes we used non-parametric statistics: the Kruskal-Wallis test to assess overall differences among the three groups, and the Wilcoxon rank sum test for independent samples to check the differences between the schizophrenia group and each of the other two comparison groups. All analyses were carried out using the Statistical Analysis System (SAS) version 6.12.

\section{RESULTS}

The mean activity for each region is shown in Table 2. In the comparison among the 
groups, we found a significant effect of the diagnosis on the left side $\left(\chi^{2}=9.54\right.$, d.f. $=2$; $P=0.008$, Kruskal-Wallis test). We found the same tendency on the right side $\left(\chi^{2}=5.60\right.$, d.f. $=2 ; P=0.06$, Kruskal-Wallis test). The schizophrenia group showed hypofrontality in comparison with the control group in the Wilcoxon test $(Z=-2.51$, $P=0.01$, on the left side). The schizophrenia group also demonstrated hypofrontality compared with the non-schizophrenia group in the Wilcoxon test $(Z=-2.21$, $P=0.03$, on the left side; $Z=-2.50$, $P=0.01$, on the right side) (Fig. 2). There was no difference in the metabolic activity values of the occipital cortex among the three groups $\left(\chi^{2}=0.52\right.$, d.f. $=2, P=0.78$; $\chi^{2}=3.47$, d.f. $=2 ; P=0.18$, respectively for the left and right sides, Kruskal-Wallis test).

\section{DISCUSSION}

\section{Hypofrontality in early stages of schizophrenia}

Our results, obtained in a male sample population, agree with the hypothesis that people with a first episode of schizophrenia demonstrate hypofrontality during the performance of an attention task; people with acute psychosis who do not progress to schizophrenia do not demonstrate this anomaly. This finding fits with the neurodevelopment theories of schizophrenia, which postulate the existence of a set of alterations that appear to be present even in the earliest phases of the disease. This hypofrontality in the initial stages of firstepisode schizophrenia is similar to that found in treatment-naïve patients and those with chronic schizophrenia (Buchsbaum

Table 2 Metabolic activity values (per $\mathrm{ml}$ ) for each group in the dorsolateral prefrontal and occipital regions (significant differences in comparison of the values of the schizophrenia group $v$. the control and nonschizophrenia groups by a Wilcoxon rank sum test for two independent samples are marked with asterisks)

\begin{tabular}{lccc}
\hline Cortical region & $\begin{array}{c}\text { Schizophrenia } \\
\text { group }(n=6) \\
\text { Mean (s.d.) }\end{array}$ & $\begin{array}{c}\text { Non-schizophrenia } \\
\text { group }(n=7) \\
\text { Mean (s.d.) }\end{array}$ & $\begin{array}{c}\text { Control } \\
\text { gorup }(n=8) \\
\text { Mean (s.d.) }\end{array}$ \\
\hline Left DLPF & $93.5(4.4)$ & $102.1(4.5)^{* *}$ & $102.8(7.7)^{* *}$ \\
Right DLPF & $99.2(3.2)$ & $105.8(5.2)^{*}$ & $105.0(6.3)$ \\
Left occipital & $104.0(5.7)$ & $102.4(2.7)$ & $104.9(5.3)$ \\
Right occipital & $104.3(6.0)$ & $100.0(3.0)$ & $103.4(3.4)$ \\
\hline
\end{tabular}

DLPF, dorsolateral prefrontal.

$* P<0.05 ; * * P<0.01$.
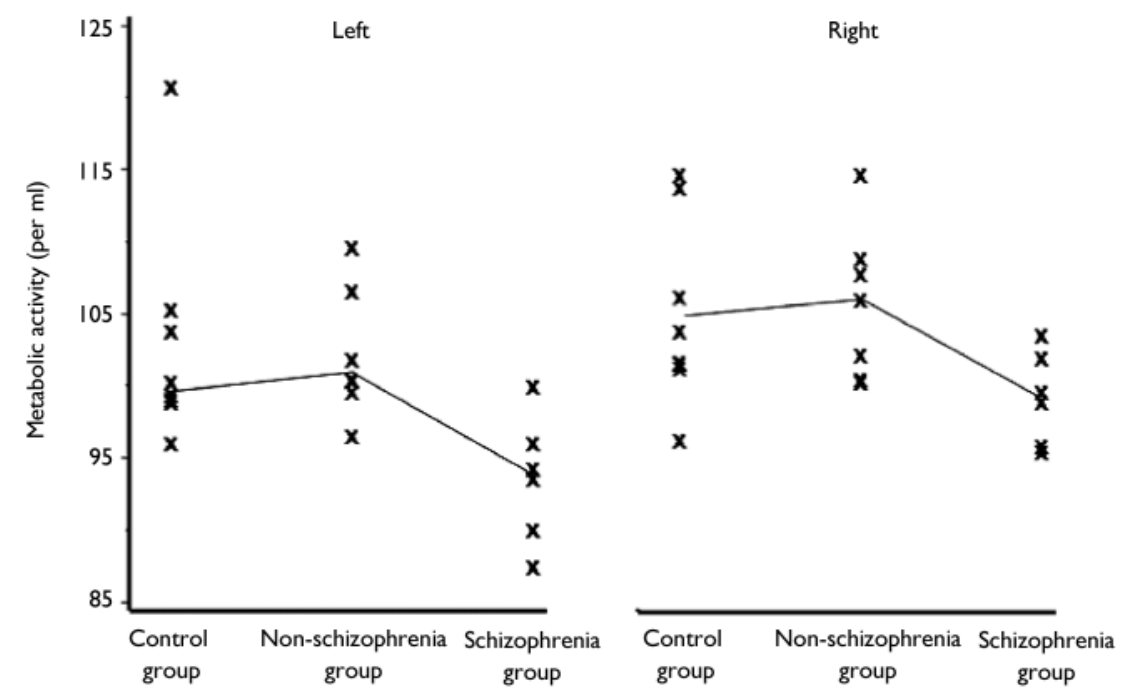

Fig. 2 Raw values of metabolic activity for the left and right dorsolateral prefrontal cortex in patients and controls. Mean values for each group are shown linked. Values for the schizophrenia group $(n=6)$ were significant in the left and right dorsolateral prefrontal cortex compared with the non-schizophrenia group $(n=7)$ and also in the left dorsolateral prefrontal cortex when compared with the control group $(n=8)$.

et al, 1992; Schroeder et al, 1994). Although the participants in our schizophrenia group were slightly younger than those in the comparison groups, their hypofrontality cannot be attributed to age differences, since there was no association between age and activity in our sample. Besides, cortical metabolic activity in the normal population declines with age (Marchal et al, 1992).

\section{Pharmacological effects on hypofrontality}

The hypofrontality found in this study does not seem to be attributable to pharmacological effects, since both patient groups had received the same treatment before the PET scan. The higher activity in the dorsolateral prefrontal region in the psychosis patients without schizophrenia could be tentatively attributed to poorer treatment compliance by these patients than by those with schizophrenia. However, the nursing personnel carefully verified this aspect and the haloperidol was administered as a liquid formulation, so lack of compliance may reasonably be ruled out. On the other hand, there is no reason a priori to expect better treatment compliance from the schizophrenia group than from the non-schizophrenia group of patients. Also, in the event of better treatment compliance by the schizophrenia group, we should have observed a corresponding change in the metabolic activity of the occipital lobe (Bartlett et al, 1994), which was not the case (Table 2).

A different treatment response in each of the patient groups might explain the lower dorsolateral prefrontal cortical activity in the people who went on to develop schizophrenia than in the non-schizophrenia patient group. This possibility was suggested by a previous study (Bartlett et al, 1998), which showed that patients who did not respond to treatment demonstrated more marked changes in activity in this brain area than responders. This does not seem to be the case in our sample, since patients in our schizophrenia group did, in fact, respond to treatment, except for negative symptoms (Table 1). In addition, other studies have shown the same hypofrontal pattern in completely drug-free patients (Buchsbaum et al, 1992; Andreasen et al, 1997). On the other hand, if the hypofrontality were caused by differences in response or in compliance with haloperidol treatment, this would not 
explain the absence of differences in the occipital cortex, since the aforesaid decrease in activity - observed solely in the nonresponders - was generalised in the cortex (Bartlett et al, 1998). Finally, the Scottish Schizophrenia Research Group (Anonymous, 1998) reported the existence of hypofrontality prior to initiation of treatment and its persistence after 6 months of treatment. Analysed together, these data support the fact that no treatment-related factor would explain the differences between our patient groups.

\section{Hypofrontality in other types of psychosis}

There is little information on cerebral activity in schizophreniform psychoses with good prognosis. Nevertheless, clinical data support the theory that cerebral substrates may differ between schizophreniform and schizophrenic psychoses; for example, in a recent study persistent primary symptoms were not found in 61 people with schizophreniform psychosis, whereas these symptoms were found in $26 \%$ of 358 people with schizophrenia (Peralta \& Cuesta, 2004). However, there are several studies of bipolar disorder, which also may commence clinically with first-episode psychosis and has a better prognosis than schizophrenia. There is evidence of decreased dorsolateral prefrontal metabolic activity under activation conditions (word generation and decision-making) during manic episodes (Blumberg et al, 1999; Rubinsztein et al, 2001), although these studies were not performed in the initial stages of the disease, and participants were receiving various medications. In the case of bipolar mania, hypoactivation may be right-lateralised (Blumberg et al, 1999; Rubinsztein et al, 2001) and accompanied by increased activity in the caudate and cingulate regions (Blumberg et al, 2000; Rubinsztein et al, 2001). These findings are different from the pattern observed not only in our patients with first-episode schizophrenia but also in untreated and in treated patients (Siegel et al, 1993; McGuire et al, 1998; Shihabuddin et al, 1998), suggesting that a degree of caution is essential before considering hypofrontality to be a specific marker for schizophrenia. In particular, our results do not support the supposition that hypofrontality may be a specific marker of schizophrenia, but only suggest that it could distinguish between first psychotic episodes of good prognosis and first episodes evolving to schizophrenia.

\section{Methodological issues}

Our study has various limitations. One of them is the small sample size. However, the short duration of the disease and the prospective diagnosis in patients with first-episode psychosis worked in our favour. Only a prospective follow-up can separate recent-onset schizophrenia from other psychotic episodes. This approach offers the advantage of studying the patients very early, as cerebral changes due to the disease may take place even in the first months (Gur et al, 1998; Rapoport et al, 1999; Pantelis et al, 2003). Besides, our patients were not exposed to other potentially brain-damaging situations, such as drug misuse or severe behaviour disorganisation. In consequence, although our sample is small, it should offer greater homogeneity than other studies using a cross-sectional design on a sample of people with first psychotic episodes. Another problem is that the brief treatment administered could hinder interpretation of the results, even though it did allow the study of a group that was truly representative of the usual presentation of acute schizophrenia. Neuroimaging studies could easily be biased towards cases in which the patient is likely to cooperate well with the procedures, which might lead to certain types of patients being more strongly represented in the sample. This problem was partially overcome by administering the same treatment to all patients prior to the PET scans. Concerning the specificity of our performance task for the frontal area of the brain, we did not perform a control task to rule out non-frontal aspects, as it would have complicated the FDG protocol significantly. Nevertheless, despite this lack of a control reference, the frontal involvement related to our performance task seems unquestionable (Rosvold et al, 1956). Finally, our findings cannot be directly generalised to women with schizophrenia, because of the greater number of structural anomalies in male patients (Nopoulos et al, 1997).

Other groups have also enrolled patients recently diagnosed with schizophrenia or schizophreniform disorder (Rubin et al, 1991). Our study group has the advantage of the prospective followup, which allowed us to differentiate between patients who were subsequently diagnosed as having schizophrenia and those who were not. In conclusion, our data suggest that the existence of hypofrontality could contribute to predicting which patients with a first episode of psychosis are likely to progress to schizophrenia.

\section{ACKNOWLEDGEMENTS}

The authors thank Dr M. A. Pozo and Dr A. Maldonado from the Centro PET Complutense for their advice and collaboration in performing the PET scans. The study was supported in part by grants from Fondo de Investigaciones Saniterias (02/1178, 02/3095, Red Temática IM3), Ministerio de Ciencia y Tecnología TIC (2001-3697-C03-03) and Fundación La Caixa (99/042-00).

\section{REFERENCES}

American Psychiatric Association (1994) Diagnostic and Statistical Manual of Mental Disorders (4th edn) (DSM-IV). Washington, DC: APA.

Andreasen, N. C., Rajarethinam, R., Cizadio, T., et al (1996) Automatic atlas-based volume estimation of human brain regions from MR images. Journal of Computer Assisted Tomography, 20, 98-106.

Andreasen, N. C., O'Leary, D. S., Flaum, M., et al (1997) Hypofrontality in schizophrenia: distributed dysfunctional circuits in neuroleptic-naïve patients. Lancet, 349, 1730-1734.

Anonymous (1998) Regional cerebral blood flow in first-episode schizophrenia patients before and after antipsychotic drug treatment. Scottish Schizophrenia Research Group. Acta Psychiatrica Scandinavica, 97, 440-449.

Ashburner, J. \& Friston, K. J. (1997) Multimodal image coregistration and partitioning - a unified framework. Neuroimage, 6, 209-217.

Bartlett, E. J., Brodie, J. D., Simkowitz, P., et al (1994) Effects of haloperidol challenge on regional cerebral glucose utilization in normal human subjects. American Journal of Psychiatry, I5I, 68I-686.

Bartlett, E. J., Brodie, J. D., Simkowitz, P., et al (1998) Effect of a haloperidol challenge on regional brain metabolism in neuroleptic-responsive and nonresponsive schizophrenic patients. American Journal of Psychiatry, I55, 337-343.

Blumberg, H. P., Stern, E., Ricketts, S., et al (1999) Rostral and orbital prefrontal cortex dysfunction in the manic state of bipolar disorder. American Journal of Psychiatry, 156, 1986-1988

Blumberg, H. P., Stern, E., Martinez, D., et al (2000) Increased anterior cingulate and caudate activity in bipolar mania. Biological Psychiatry, 48, 1045-1052.

Buchsbaum, M. S., Haier, R. J., Potkin, S. G., et al (1992) Frontostriatal disorder of cerebral metabolism in never-medicated schizophrenics. Archives of General Psychiatry, 49, 935-942.

Desco, M., Pascau, J., Reig, S., et al (200I) Multimodality image quantification using Talairach grid. In Proceedings of SPIE Medical Imaging, vol. 4332, pp. 1385-1392. San Diego, CA: IEEE.

First, M. B., Spitzer, R. L., Gibbon, M., et al (1997) Structured Clinical Interview for DSM-IV Axis I Disorders - Clinician Version. Washington, DC: American Psychiatric Association. 
Frackowiak, R. S. J., Friston, K. J., Frith, C. D., et al (1997) Human Brain Function. San Diego, CA: Academic Press.

Gur, R. E., Cowell, P., Turetsky, B. I., et al (1998) A follow-up magnetic resonance imaging study of schizophrenia. Relationship of neuroanatomical changes to clinical and neurobehavioral measures. Archives of General Psychiatry, 55, 145-152.

Hollingshead, A. \& Frederick, R. (1953) Socia stratification and psychiatric disorders. American Sociological Review, 18, 163-189.

Kates, W. R., Worsofsky, I. S., Patwardhan, A., et al (1999) Automated Talairach atlas-based parcellation and measurement of cerebral lobes in children. Psychiatry Research: Neuroimaging, 9I, II-30.

Kay, S. R., Opler, L. A. \& Fiszbein, A. (1992) Positive and Negative Syndrome Scale (PANSS): Manual. New York: Multi-Health Systems.

Marchal, G., Rioux, P., Petit-Taboue, M. C., et a (1992) Regional cerebral oxygen consumption, blood flow, and blood volume in healthy human aging. Archives of Neurology, 49, 1013-1020.

McGuire, P. K., Quested, D. J., Spence, S. A., et a (1998) Pathophysiology of 'positive' thought disorder in schizophrenia. British Journal of Psychiatry, 173, 231-235.

Molina, V., Reig, S., Sarramea, F., et al (2003) Anatomical and functional brain variables associated with clozapine response in treatment-resistant schizophrenia. Psychiatry Research: Neuroimaging, 124 $|53-16|$.

Nopoulos, P., Flaum, M. \& Andreasen, N. C. (1997) Sex differences in brain morphology in schizophrenia. American Journal of Psychiatry, I54, 1648-1654.

Pantelis, C., Velakoulis, D., McGorry, P. D., et al (2003) Neuroanatomical abnormalities before and after onset of psychosis: a cross-sectional and longitudinal MRI comparison. Lancet, 361, 28I-288.

Peralta, V. \& Cuesta, M. J. (2004) The deficit syndrome of the psychotic illness. A clinical and nosological study. European Archives of Psychiatry and Clinical Neurosciences, 254, 165-17

Rapoport, J. L., Giedd, J. N., Blumenthal, J., et a (1999) Progressive cortical change during adolescence in childhood-onset schizophrenia. A longitudinal magnetic resonance imaging study. Archives of General Psychiatry, 56, 649-654

Rosvold, H., Mirsky, A., Sarason, I., et al (1956) A continuous-performance test of brain damage. Journal of Consulting Psychology, 20, 343-352.

Rubin, P., Holm, S., Friberg, L., et al (1991) Altered modulation of prefrontal and subcortical brain activity in newly diagnosed schizophrenia and schizophreniform disorder. A regional cerebral blood flow study. Archives of General Psychiatry, 48, 987-995.

Rubinsztein, J. S., Fletcher, P. C., Rogers, R. D., et al (200I) Decision-making in mania: a PETstudy. Brain, I24, 2550-2563.

\section{CLINICAL IMPLICATIONS}

- Hypofrontality seems to be a marker of schizophrenia even at the time of the patient's first psychotic episode.

- If the predictive value of hypofrontality is confirmed, decisions about the most suitable treatment will be facilitated.

- The presence of hypofrontality in first-episode psychosis fits with the neurodevelopmental theories of schizophrenia.

\section{LIMITATIONS}

Our results are limited to a male population. Conclusions should be confirmed in a larger sample size including both men and women.

- The brief treatment administered might have affected our results. However, the possible confounding effect should be minimal because both groups of patients received the same treatment.

- Other forms of psychosis, such as bipolar disorder or psychotic depression, may also show hypofrontality at early stages.

V. MOLINA, PhD, Department of Psychiatry, Hospital Cliníco Universitario de Salamanca; J. SANZ, MD, Department of Psychiatry, Hospital Doce de Octubre, Madrid; S. REIG, PhD, R. MARTíNEZ, PhD, Department of Experimental Medicine, Hospital Gregorio Marañón, Madrid; F. SARRAMEA, PhD, R. LUQUE, MD Department of Psychiatry, Hospital Reina Sofía, Còrdoba; C. BENITO, MD, Clínica Monterprinciple, Hospital de Madrid; D. GISPERT, PhD, J. PASCAU, MSc, M. DESCO, PhD, Department of Experimental Medicine, Hospital Gregorio Marañón, Madrid, Spain

Correspondence: Dr V. Molina, Department of Psychiatry, Hospital Clínico de Salamanca, Paseo de San Vicente, 58-182, 37007b Salamanca, Spain. Tel: +34 923 29|383; Fax: +34 923 229158; e-mail: vmolina@usal.es

(First received I5 December 2003, final revision 23 September 2004, accepted 30 September 2004

Schroeder, J., Buchsbaum, M. S., Siegel, B.V., et al (1994) Patterns of cortical activity in schizophrenia. Psychological Medicine, 24, 947-955.

Shihabuddin, L., Buchsbaum, M. S., Hazlett, E. A. et al (1998) Dorsal striatal size, shape, and metabolic rate in never-medicated and previously medicated schizophrenics performing a verbal learning task. Archives of General Psychiatry, 55, 235-243.

Siegel, B.V., Buchsbaum, M. S., Bunney, W. E., et a (1993) Cortical-striatal-thalamic circuits and brain glucose metabolic activity in 70 unmedicated male schizophrenic patients. American Journal of Psychiatry, 150, 1325-1336.
Talairach, J. \& Tournoux, P. (1988) Co-Planar Stereotaxic Atlas of the Human Brain. New York: Thieme.

Volz, H., Gaser, C., Häger, F., et al (1999) Decreased frontal activation in schizophrenics during stimulation with the continuous performance test - a functional magnetic resonance imaging study. European Psychiatry, 14, 17-24.

Woods, R. P., Mazziotta, J. C. \& Cherry, S. R. (1993) MRI-PET registration with automated algorithm. Journal of Computer Assisted Tomography, 17, 536-546. 